\title{
Copula duration and sperm economy in the large thistle aphid, Uroleucon cirsii (Hemiptera: Aphididae)
}

\author{
JOACHIM L. DAGG \\ Institute of Phytopathology and Plant Protection, Grisebachstr. 6, University of Göttingen, 37077 Göttingen, Germany; e-mail: \\ jdagg@gwdg.de
}

Key words. Uroleucon cirsii, Scatophaga (syn. Scathophaga) stercoraria, mating behaviour, copula duration, sperm economy, mate choice

\begin{abstract}
Experiments on copula duration indicate that the nature of mate competition and sperm storage in the large thistle aphid, Uroleucon cirsii (L.), differs from that in the well-studied yellow dung fly, Scatophaga stercoraria (L.). Unlike the situation in the dung fly, second copulas are shorter on average than first copulas for both individual males and females. This suggests that male aphids have a limited amount of sperm at their disposal and that the females capacity to store sperm is limited as could be expected when males were not able to displace sperm from former copulas.
\end{abstract}

\section{INTRODUCTION}

Optimal mating theory has been pioneered by Geoffrey Parker and colleagues (e.g. Parker, 1970; Parker \& Stuart, 1976) who studied the yellow dung fly, Scatophaga stercoraria (L.). In this system, "sperm economy" (i.e. the production, storage, and use of sperm) is characterised by four factors: 1) sperm is not a limiting factor for males, they can always invest more if necessary; 2) newly transferred sperm instantly mixes with the contents of the female's storage organ; 3 ) each amount of newly transferred sperm displaces an equal amount of fluid or stored sperm from the female's storage organ; 4) the sperm of the last male to mate gains precedence in fertilising the eggs of the next batch (e.g. Parker et al., 1990; Parker \& Simmons, 1991; Simmons et al., 1999). Thus the risk of sperm displacement rises with the portion of a male's sperm in the store. This model predicts a copula duration which ensures that the last male to mate with a female will fertilise about $80 \%$ of the eggs laid in the next batch, which has been validated by experimental results (Parker, 1970; Parker \& Stuart, 1976; Birkhead \& Parker, 1997). As a consequence of sperm displacement a male will copulate as long, on average, with a virgin female as with one that has just been mated.

Later research on yellow dung flies has become more finegrained and differential effects of characters like body size have been studied. Males that are larger than average will copulate for a shorter time, because they can gain more females through take-overs and their rate of sperm transfer and thus sperm displacement is higher than for smaller males (e.g. Parker \& Simmons, 1994; Charnov \& Parker, 1995; Birkhead \& Parker, 1997). The size of the female has also been found to be important (e.g. Charnov \& Parker, 1995; Parker et al., 1999).

Without size differences, however, the duration of subsequent copulas remains constant. Constant average copula duration can be taken as circumstantial evidence for the nature of sperm competition in $S$. stercoraria. If, in another species, subsequent copulas became longer or shorter, the storage and use of sperm should somehow differ from that in S. stercoraria. This has been tested for an aphid species.

\section{MATERIAL AND METHODS}

Uroleucon cirsii (L.) is a large aphid species $(4-5.2 \mathrm{~mm})$ that lives on the leaves and stems of some thistle species, e.g. Cir- sium oleraceum (L.) or C. arvense (L.), but does not alternate between hosts. That is, it occurs on a range of species throughout the year, but does not have special summer and winter hosts. Parthenogenetically over-wintering lineages are not known for this species. Seeds of $C$. arvense and parthenogenetic females of $U$. cirsii were collected in 1999 on the campus of the University of East Anglia in Norwich, and a greenhouse culture was established and held under long day conditions (18h light, $20^{\circ} \mathrm{C}$ ). While the animals for this culture came from one field colony, the possibility that they stemmed of more than one clone cannot be entirely excluded. Parthenogenetic females from this culture were transferred to short-day conditions $(12 \mathrm{~h}$ light, $15^{\circ} \mathrm{C}$ ). The sexual animals of this species can be easily discriminated, because the males are born leaf green and the females chocolate brown (pictures demonstrating that this is a qualitative and striking difference are available on request). The sexual females and males were separated as new-born nymphs and transferred to potted plants under cylindrical cages $(30 \mathrm{~cm}$ high with gauze sealed holes for ventilation) into separated but identical climatic cabinets. Thus, animals chosen for experimentation had not previously mated.

Mating was observed on leaves, the petioles of which were inserted into watered blocks of floral foam inside plastic boxes $(7.5 \times 4.5 \times 2 \mathrm{~cm})$, which had a hole covered with gauze for ventilation. Mating behaviour was observed through the front window of a climatic cabinet at $15^{\circ} \mathrm{C}$ and a light intensity of 93 $\mu \mathrm{E} \cdot \mathrm{m}^{-2} \mathrm{~s}^{-1}$ at the level of the plastic boxes. Copula duration was measured with a hand-held stop watch. All observations were made between 90 and 210 minutes after the lights came on. Virgin males and females were chosen at random for the following experimental groups.

\section{Group 1: One male with different females}

One male was placed together with 5 females. Males that did not mate within half an hour were discarded and further observation was broken off if no second mating occurred within half an hour after the first one. The duration of the first and second copulation of a male with females were recorded. Events of double mating with the same female were excluded from the analysis. This was repeated until 46 instances of one male mating with two different females had been observed. 


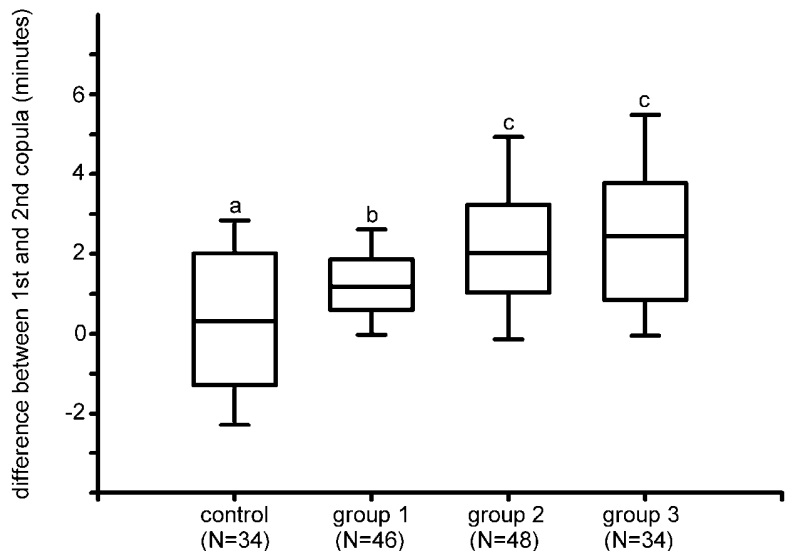

Fig. 1. Box-and-whiskers plots for the difference in duration between the first and second copula. Boxes cover 50 percent of the variance from the 25 th to the 75 th percentile and are divided at the median. The whiskers stretch to the 10th and 90th percentile respectively. The control was sampled from a normal distribution with a mean of zero and a standard deviation of 2.33 (median $=0.32$ ). Group 1: One male had access to five females (median $=1.18 \mathrm{~min})$. Group 2: Five males had access to one female (median $=2.02 \mathrm{~min})$. Group 3: One male had access to one female (median $=2.45 \mathrm{~min}$ ). Different letters mark significantly different samples.

\section{Group 2: One female with different males}

A female was placed in a box containing 5 males. Females that were not mated within half an hour of the start of the observation were discarded and further observation was broken off if no second mating occurred within half an hour after the first one. The time spent in copulation by the first and second male was recorded. Events of double mating with the same male were excluded from the analysis. This was repeated until 48 instances of one female mating with two different males had been observed.

\section{Group 3: Single pairs allowed to mate twice}

A male and a female were placed in a standard box. Pairs that were reluctant to mate were discarded and further observation was broken off if no second mating occurred within half an hour after the first one. The duration of two copulas was recorded This was repeated until 34 instances of one male mating twice with the same female had been observed.

\section{Statistical analysis}

The differences in copula duration (1st-2nd) were tested against one another. The Kruskal-Wallis-test followed by pairwise comparisons of the Mann-Whitney-Wilcoxon U-statistic was chosen as the non-parametric alternative to an ANOVA. A normal distribution with a mean of zero and a standard deviation equal to the highest one among the experimental groups ( $\mathrm{SD}=$ 2.33) served as a control population, from which a random sample of a size equal to the smallest one among the experimental groups ( $\mathrm{N}=34$ values) was drawn as the control sample. This control sample happened to have a mean of 0.11 and a standard deviation of 2.18. It represents a result from a population with equal duration of first and second copula.

Missing values arose from instances where no second mating occurred within half an hour after the end of the first mating. The events of a second copula or no second copula respectively can be taken as two distinct outcomes of the experiments. These categorical data were compared with Fisher's exact test for each pair of experimental groups $(1-2,2-3,3-1)$.
TABLE 1. Non-parametric pair-wise comparisons.

\begin{tabular}{lcccc}
\hline Comparison & U-statistic & p-values & $\alpha$ & $\mathrm{K}$ \\
\hline control-group1 & 543.0 & $0.020^{*}$ & 0.0253 & 2 \\
control-group2 & 405.0 & $<0.001^{*}$ & 0.0085 & 6 \\
control-group3 & 253.0 & $<0.001^{*}$ & 0.0102 & 5 \\
group1-group2 & 720.5 & $0.004^{*}$ & 0.0127 & 4 \\
group1-group3 & 1065.5 & $0.006^{*}$ & 0.0169 & 3 \\
group2-group3 & 871.5 & 0.601 & 0.0500 & 1 \\
\hline
\end{tabular}

P-values with asterisk are significant at the $\alpha^{\prime}$ levels associated to $\mathrm{a}=0.05$ as calculated by the Dunn-Sidák method (cf. Sokal \& Rohlf 1995, p. 239): $\alpha^{\prime}=1-(1-\alpha)^{1 / K}$, where $\mathrm{K}$ is the rank of the p-value.

\section{RESULTS}

The duration of the second copula was consistently shorter than the duration of the first copula in all experimental groups (Fig. 1). The Kruskal-Wallis statistic had a small probability ( $\mathrm{H}$ $=25.58, \mathrm{p}<0.001,3 \mathrm{df}$ ). The results of the pair-wise comparisons are summarised in Table 1. Copula duration reduced significantly between the first and second mating in all three groups. With one male mating twice, this reduction was significantly weaker than with one female mating twice. Pairs mating twice showed the strongest reduction, but this was not significantly different from one female mating with different males.

Second copula events were significantly more common, when one male had access to different females in group 1 than vice versa in group 2 (Pearson's Chi-square $=6.091 ; 1 \mathrm{df}$; Fisher's exact test: $\mathrm{p}=0.019)$. Again, second copulas were more common in group 2 (1female : 5males) than in group 3 (Pearson's Chi-square $=8.221 ; 1 \mathrm{df}$; Fisher's exact test: $\mathrm{p}=0.005$ ). The difference between group 3 and group 1 must, of course, be still more pronounced (Pearson's Chi-square $=24.111 ; 1 \mathrm{df}$; Fisher's exact test: $\mathrm{p}<0.001)$.

\section{DISCUSSION}

The present results strongly suggest that sperm production, storage, and use by of $U$. cirsii differs from the one in the yellow dung fly, $S$. stercoraria. It is therefore necessary to ask which of the four assumptions on sperm "economy" (unlimited sperm resource of males, instant mixing in the female's store, sperm displacement from the store, last male precedence) need to be changed in order to explain the above results. The reduction of the duration of the copula of individual males (group 1) suggests a limitation in the amount of sperm a male has at its disposal. The reduction in the duration of the copula of individual females (group 2) could be expected, if males were not able to displace stored sperm. Reduced copula duration might therefore be a simple consequence of a limit on the female's capacity to store sperm.

Although the results presented here do not allow any inference about the mixing of the sperm in the female's store, first evidence suggests that aphid sperm comes in co-operating bundles with the heads of the sperm cells sticking together and the flagellae beating synchronously (cf. Dagg, 2002; appendix 1.3). Even with bundles of co-operating sperm and no sperm displacement, however, the last male's sperm could gain precedence in fertilising eggs, simply because it might be situated closer to the exit of the female's sperm store (Simmons \& SivaJothy, 1998).

The categorical analysis of second copula events revealed an effect of a previous copula on the likelihood of a second copula. 
Second copula events were most likely when one male had access to different females (group 1), less likely when different males had access to one female (group 2), and least likely when one male had access to one female (group 3). This suggests an element of mate choice in aphids, though it cannot be inferred by which sex.

ACKNOWLEDGEMENTS. My visit at the UEA in Norwich was funded by the Deutscher Akademischer Austausch Dienst. Thanks to A.F.G. Dixon and S. Vidal for support and D. Hales for comments on a former version.

\section{REFERENCES}

BirkHead T.R. \& Parker G.A. 1997: Sperm competition and mating systems. In: Krebs J.R. \& Davies N.B. (eds): Behavioural Ecology: an Evolutionary Approach, Fourth Edition. Blackwell Science, Oxford, pp. 121-145.

Charnov E.L. \& Parker G.A. 1995: Dimensionless invariants from foraging theory's marginal value theorem. Proc. Natl. Acad. Sci. USA 92: 1446-1450.

DAGG J.L. 2002: Strategies of Sexual Reproduction in Aphids. $\mathrm{Ph}$.D. thesis. University of Göttingen, Germany. [http://webdoc.sub.gwdg.de/diss/2002/dagg/dagg.pdf]
PARKer G.A. 1970: Sperm competition and its evolutionary effect on copula duration in the fly Scatophaga stercoraria. $J$. Insect Physiol. 16: 1301-1328.

PARKer G.A. \& StUART R.A. 1976: Animal behaviour as a strategy optimizer. Amer. Nat. 110: 1055-1076.

Parker G.A. \& Simmons L.W. 1991: A model of constant random sperm displacement during mating: evidence from Scatophaga. Proc. R. Soc. Lond. B 246:107-115.

Parker G.A. \& Simmons L.W. 1994: Evolution of phenotypic optima and copula duration in dungflies. Nature 370: 53-56.

PArker G.A., Simmons L.W. \& Kirk H. 1990: Analysing sperm competition data. Behav. Ecol. Sociobiol. 27: 55-65.

Parker G.A., Simmons L.W., Stockley P., McChristie D.M. \& Charnov E.L. 1999: Optimal copula duration in yellow dung flies: effects of female size and egg content. Anim. Behav. 57: 795-805.

Simmons L.W. \& Siva-Jothy M.T. 1998: Sperm competition in insects: mechanisms and the potential for selection. In: Birkhead T.R. \& Møller A.P. (eds): Sperm Competition and Sexual Selection. Academic Press, San Diego, pp. 341-434

Simmons L.W., Parker G.A. \& Stockley P. 1999: Sperm displacement in the yellow dung fly, Scatophaga stercoraria: an investigation of male and female processes. Amer. Nat. 153: 302-314.

Received May 20, 2002; revised October 14, 2002; accepted October 21, 2002 